\title{
Comparison of three molecular typing methods in studies of transmission of Porphyromonas gingivalis
}

\author{
T. J. M. VAN STEENBERGEN, C. MENARD*, C. J. TIJHOF, C. MOUTON* and J. DE GRAAFF
}

Department of Oral Microbiology, Academic Centre for Dentistry Amsterdam, Van der Boechorststraat 7, 1081 BT Amsterdam, The Netherlands and *Groupe de Recherche en Ecologie Buccale, Faculté de Medecine Dentaire, Université Laval, Quebec G1K7P4, Canada

\begin{abstract}
Summary. Porphyromonas gingivalis is associated strongly with severe periodontitis, but little information is available on possible transmission routes of this species. This study evaluated three DNA-based molecular typing methods for use in epidemiological surveys of $P$. gingivalis. In total, 32 isolates from eight married couples were investigated by: (i) restriction endonuclease analysis (REA) of whole chromosomal DNA; (ii) hybridisation of DNA fragments with ribosomal DNA (ribotyping); and (iii) amplification of DNA by the polymerase chain reaction with arbitrary primers (AP-PCR). The data obtained with the three methods were in broad agreement: in six of the eight couples, the isolates from husband and wife were indistinguishable, but isolates from unrelated individuals showed distinct types with all three methods. For some isolates, minor differences in REA pattern were obtained which could not be correlated with differences in ribotype or AP-PCR type. Ribotyping showed differences between isolates from one individual, which were indistinguishable with the other two methods. The patterns obtained with ribotyping or AP-PCR were simple in comparison to the relatively complex REA patterns. Although all three methods were concordant, AP-PCR was found to be the least time-consuming method. The data support the suggestion that $P$. gingivalis can be transmitted between spouses.
\end{abstract}

\section{Introduction}

Porphyromonas gingivalis is associated strongly with severe periodontitis, but despite much data on the prevalence, biology and virulence of this suspected pathogen, ${ }^{1-4}$ little information is available on its possible routes of transmission. We have recently provided evidence for the transmission of $P$. gingivalis between spouses. ${ }^{5}$ Similarly, although $P$. gingivalis is isolated rarely from children, Petit et al. ${ }^{6}$ have shown that when this species is found in children, one of the parents is often colonised with the same type, indicating the possibility of transmission between parents and their children.

Several typing methods have been described for epidemiological studies of $P$. gingivalis, but classical typing methods, such as biotyping and serotyping, discriminate few distinct types and are, therefore, not suitable for studying routes of transmission. Modern typing methods based on molecular biology techniques have also been described for $P$. gingivalis. These include restriction endonuclease analysis (REA) of whole chromosomal DNA, ${ }^{7-9}$ ribotyping, involving hybridisation of DNA fragments with ribosomal DNA, ${ }^{10,11}$ and amplification of DNA fragments by the poly-

Received 5 April 1993; accepted 14 May 1993 Correspondence to Dr. T. J. M. van Steenbergen. merase chain reaction with arbitrary primers (APPCR) ${ }^{12}$ The aim of the present study was to compare these three DNA-based methods for typing $P$. gingivalis.

\section{Materials and methods}

\section{Bacterial isolates}

The $P$. gingivalis isolates were obtained from the subgingival plaque, saliva and oral mucous membranes of eight patients with severe adult periodontitis, and their spouses, all colonised with $P$. gingivalis. The clinical and microbiological characteristics of these married couples have been described previously. ${ }^{5}$ Between one and seven isolates were evaluated from each individual patient or spouse.

\section{Restriction endonuclease analysis ( $R E A)$}

Chromosomal DNA was isolated by phenol: chloroform extraction and ethanol precipitation after lysis of the bacterial cells with lysozyme, proteinase $\mathrm{K}$ and SDS, as described previously. ${ }^{5}$ Samples $(2 \mu \mathrm{g})$ of DNA were digested to completion ( $2 \mathrm{~h}$ ) with Pst $\mathrm{I}$ or BamHI (Boehringer Mannheim) according to the manufacturer's recommendations. DNA fragments 
Table. Comparison of results obtained by restriction endonuclease analysis (REA), polymerase chain reaction with arbitrary primer (AP-PCR), and ribotyping for $P$. gingivalis isolates from periodontitis patients and their spouses

\begin{tabular}{|c|c|c|c|c|c|c|c|}
\hline \multirow[b]{2}{*}{ Isolate } & \multirow{2}{*}{$\begin{array}{c}\text { Couple } \\
\text { no. }\end{array}$} & \multirow[b]{2}{*}{ Subject } & \multirow[b]{2}{*}{ Site } & \multirow{2}{*}{$\begin{array}{l}\text { REA } \\
\text { type }\end{array}$} & \multirow{2}{*}{$\begin{array}{c}\text { AP-PCR } \\
\text { type }\end{array}$} & \multicolumn{2}{|c|}{ Ribotype } \\
\hline & & & & & & $B g / \mathbf{I}$ & BamHI \\
\hline HG 1293 & 1 & Patient & Pocket & A & a & 1 & I \\
\hline HG 1296 & 1 & Spouse & Pocket & B & $\mathrm{b}$ & 2 & II \\
\hline HG 1301 & 2 & Patient & Pocket & $\mathrm{C}$ & $\mathrm{c}$ & 3 & III \\
\hline HG 1306 & 2 & Spouse & Pocket & $\mathrm{C}$ & c & 3 & III \\
\hline HG 1312 & 3 & Patient & Pocket & $\mathrm{D}^{\prime}$ & $\mathrm{d}$ & 4 & IV \\
\hline HG 1310 & 3 & Patient & Saliva & D & $\mathrm{d}$ & 4 & IV \\
\hline HG 1316 & 3 & Spouse & Pocket & $\mathrm{D}^{\prime}$ & $\mathrm{d}$ & 4 & IV \\
\hline HG 1319 & 3 & Spouse & Pocket & $\mathrm{D}$ & $\mathrm{d}$ & 4 & IV \\
\hline HG 1321 & 4 & Patient & Pocket & $\mathrm{F}$ & $\mathrm{f}$ & 5 & $\mathrm{~V}$ \\
\hline HG 1326 & 4 & Spouse & Pocket & $\mathrm{F}$ & $\mathrm{f}$ & 5 & V \\
\hline HG 1331 & 5 & Patient & Pocket & $\mathrm{G}$ & $\mathrm{g}$ & 6 & VI \\
\hline HG 1332 & 5 & Patient & Pocket & $\mathrm{G}$ & $\mathrm{g}$ & 6 & VI \\
\hline HG 1333 & 5 & Patient & Tongue & $\mathrm{G}$ & $\mathrm{g}$ & 6 & VI \\
\hline HG 1334 & 5 & Patient & Buccal & G & $\mathrm{g}$ & 6 & VI \\
\hline HG 1335 & 5 & Patient & Tonsils & G & g & 6 & VI \\
\hline HG 1336 & 5 & Patient & Tonsils & $\mathrm{G}$ & $\mathrm{g}$ & 6 & VI \\
\hline HG 1337 & 5 & Patient & Saliva & G & $\mathrm{g}$ & 6 & VI \\
\hline HG 1338 & 5 & Spouse & Pocket & G & $\mathrm{g}$ & 6 & VI \\
\hline HG 1343 & 6 & Patient & Pocket & $\mathrm{H}$ & $\mathrm{h}$ & 7 & VII \\
\hline HG 1347 & 6 & Spouse & Pocket & $\mathbf{J}$ & $\mathrm{j}$ & 8 & VIII \\
\hline HG 1352 & 7 & Patient & Pocket & $\mathrm{K}$ & $\mathrm{k}$ & 9 & $\mathrm{X}$ \\
\hline HG 1353 & 7 & Patient & Saliva & $\mathrm{K}$ & $\mathrm{k}$ & 10 & $\mathrm{X}$ \\
\hline HG 1354 & 7 & Patient & Tongue & K & $\mathrm{k}$ & 10 & IX \\
\hline HG 1355 & 7 & Patient & Buccal & $\mathrm{K}$ & $\mathrm{k}$ & 10 & $\mathrm{X}$ \\
\hline $\mathrm{HG} 1356$ & 7 & Spouse & Tongue & $\mathrm{K}$ & $\mathbf{k}$ & 10 & $X$ \\
\hline HG 1357 & 7 & Spouse & Buccal & K & $\mathrm{k}$ & 10 & $X$ \\
\hline HG 1358 & 7 & Spouse & Tonsils & $\mathbf{K}$ & $\mathrm{k}$ & 10 & $\mathrm{X}$ \\
\hline HG 1359 & 7 & Spouse & Saliva & $\mathrm{K}$ & k & 10 & $\mathrm{X}$ \\
\hline HG 1493 & 8 & Patient & Pocket & $\mathbf{L}^{\prime}$ & 1 & 11 & XI \\
\hline HG 1494 & 8 & Patient & Buccal & L & 1 & 11 & XI \\
\hline HG 1495 & 8 & Spouse & Pocket & $\mathrm{L}^{\prime \prime}$ & 1 & 11 & XI \\
\hline HG 1496 & 8 & Spouse & Saliva & $\mathrm{L}^{\prime \prime}$ & 1 & 11 & XI \\
\hline
\end{tabular}

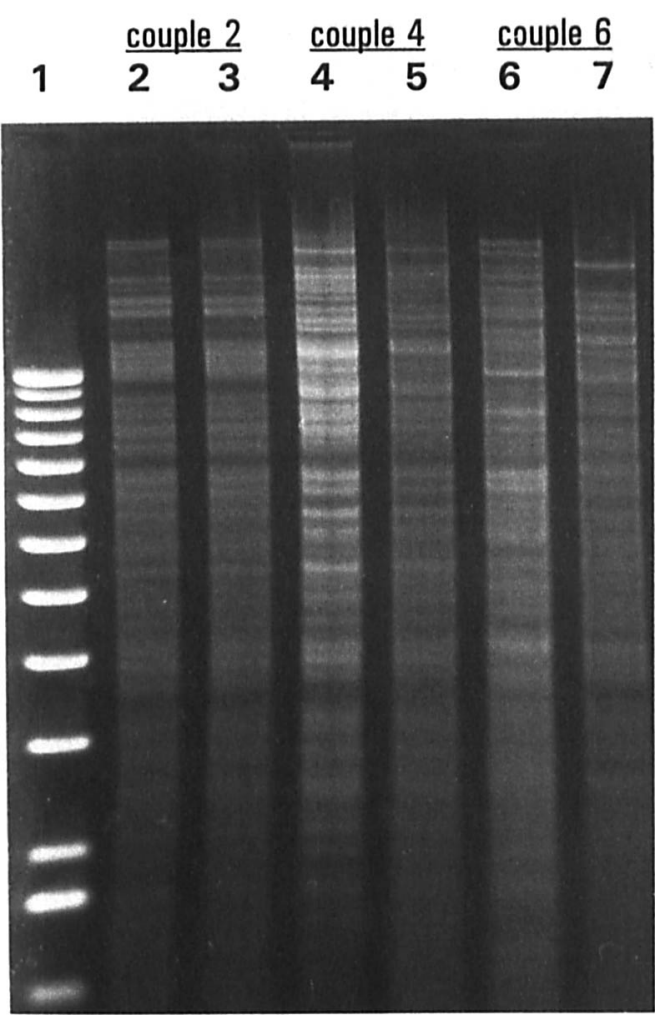

Fig. 1. BamHI restriction digests of DNA from six $P$. gingivalis isolates from three married couples. Lane 1, 1-kb DNA size marker ladder (Gibco, Gaithersburg, MD, USA); 2 and 3, couple 2, patient and spouse; 4 and 5 , couple 4 , patient and spouse; 6 and 7 , couple 6 , patient and spouse. were separated by electrophoresis on agarose $0.6 \%$ $\mathrm{w} / \mathrm{v}$ gels and stained with ethidium bromide. ${ }^{5}$

\section{Polymerase chain reaction with arbitrary primer $(A P$ - $P C R)$}

Amplification of DNA fragments in the polymerase chain reaction with arbitrary primers was as described by Menard et $\mathrm{al} .^{12}$ Briefly, one colony was harvested into $100 \mu \mathrm{l}$ of $\mathrm{H}_{2} \mathrm{O}$ with added activated charcoal, heated for $10 \mathrm{~min}$ at $100^{\circ} \mathrm{C}$, and centrifuged briefly in a microfuge; 5- $\mu$ l samples were used as template for the PCR. Two primers were used: no. 970-11 (5'GTAAGGCCG), that can detect heterogeneity in $P$. gingivalis, ${ }^{12}$ and no. 910-05 (5'-CCGGCGGCG), that has also been useful for discriminating between $P$. gingivalis strains (C. Menard and C. Mouton, unpublished results). Amplification with Taq polymerase (Pharmacia) was as described previously, ${ }^{12}$ with 30 amplification cycles. Amplification products were evaluated by electrophoresis on agarose $1.6 \% \mathrm{w} / \mathrm{v}$ gels and staining with ethidium bromide.

\section{Ribotyping}

Ribotyping was as described by Saarela et al. ${ }^{10}$ Chromosomal DNA was isolated as described for REA. The DNA was digested to completion with $B g h$ or BamHI (Boehringer Mannheim), used according to 
a
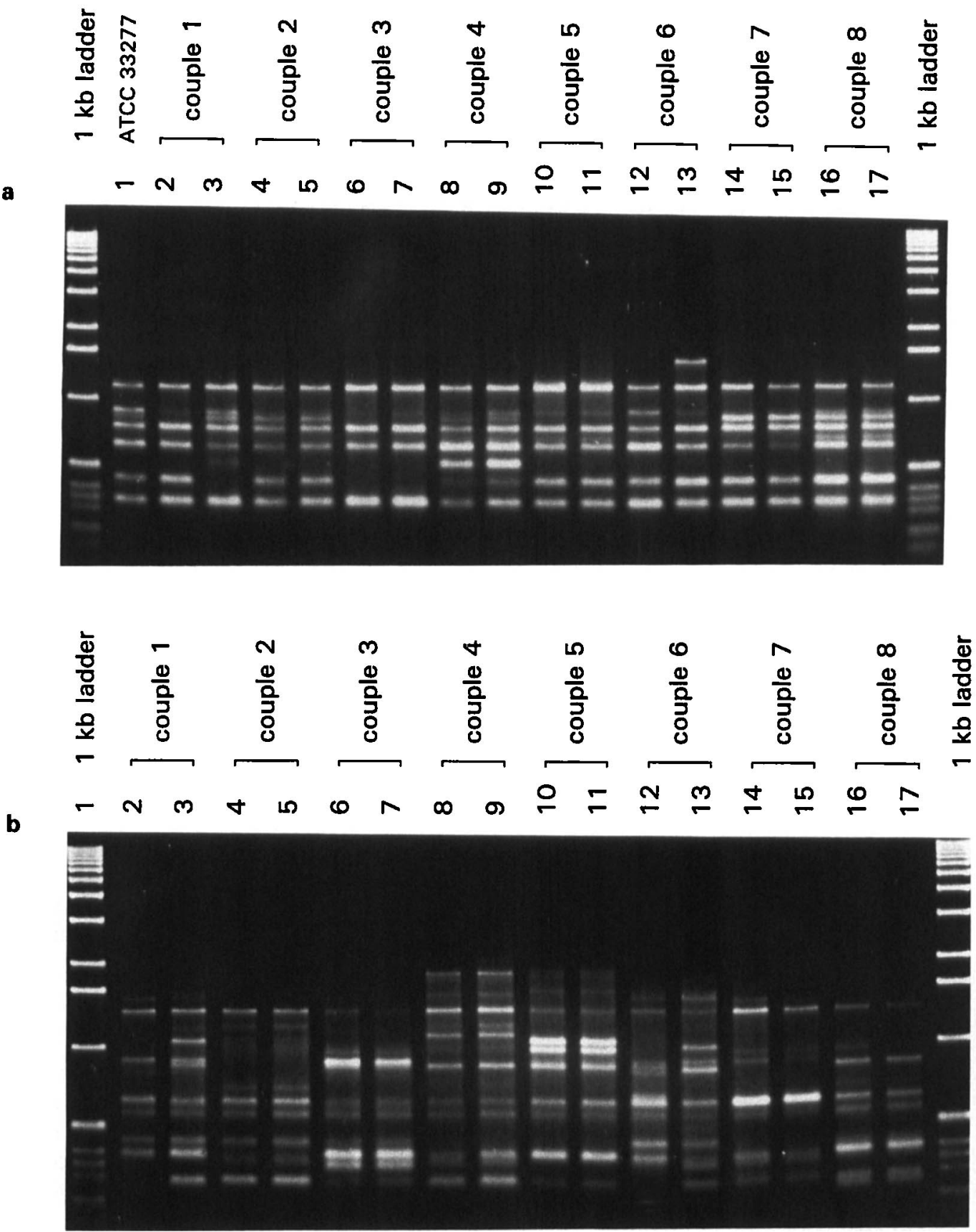

Fig. 2. Fingerprints obtained after PCR with a, primer 970-11; b, primer 910-05 and DNA from 16 P. gingivalis isolates from eight married couples.

the manufacturer's instructions. DNA fragments were separated on agarose $0.6 \% \mathrm{w} / \mathrm{v}$ gels by electrophoresis, stained with ethidium bromide, and photographed under UV illumination. Thereafter, DNA was transferred to a positively charged nylon filter (Boehringer Mannheim), fixed on to the membrane by UV illumination for $3 \mathrm{~min}$, and hybridised for $16 \mathrm{~h}$ at $68^{\circ} \mathrm{C}$ with a probe consisting of digoxigenin-labelled pKK3535 DNA. This plasmid contains the rDNA operon from Escherichia coli, ${ }^{13}$ and was obtained from M. Saarela, Helsinki, Finland. The plasmid was isolated from a strain of $E$. coli by the Magic Miniprep procedure (Promega, Madison, WI, USA), and was then labelled with digoxigenin (Boehringer Mannheim) according to the manufacturer's instructions.
Hybridised fragments were detected by incubation with anti-digoxigenin antibodies labelled with alkaline phosphatase, followed by incubation with nitro-bluetetrazolium, or with the luminescent substrate Lumigen-PPD (Boehringer Mannheim). As detection with Lumigen-PPD gave the clearest bands, this procedure was used in most experiments.

\section{Results}

Three different methods (REA typing, ribotyping and AP-PCR) for typing $P$. gingivalis were compared for a total of 32 isolates obtained from 16 individuals (table). These isolates were chosen as being representative of the different REA types that were found 


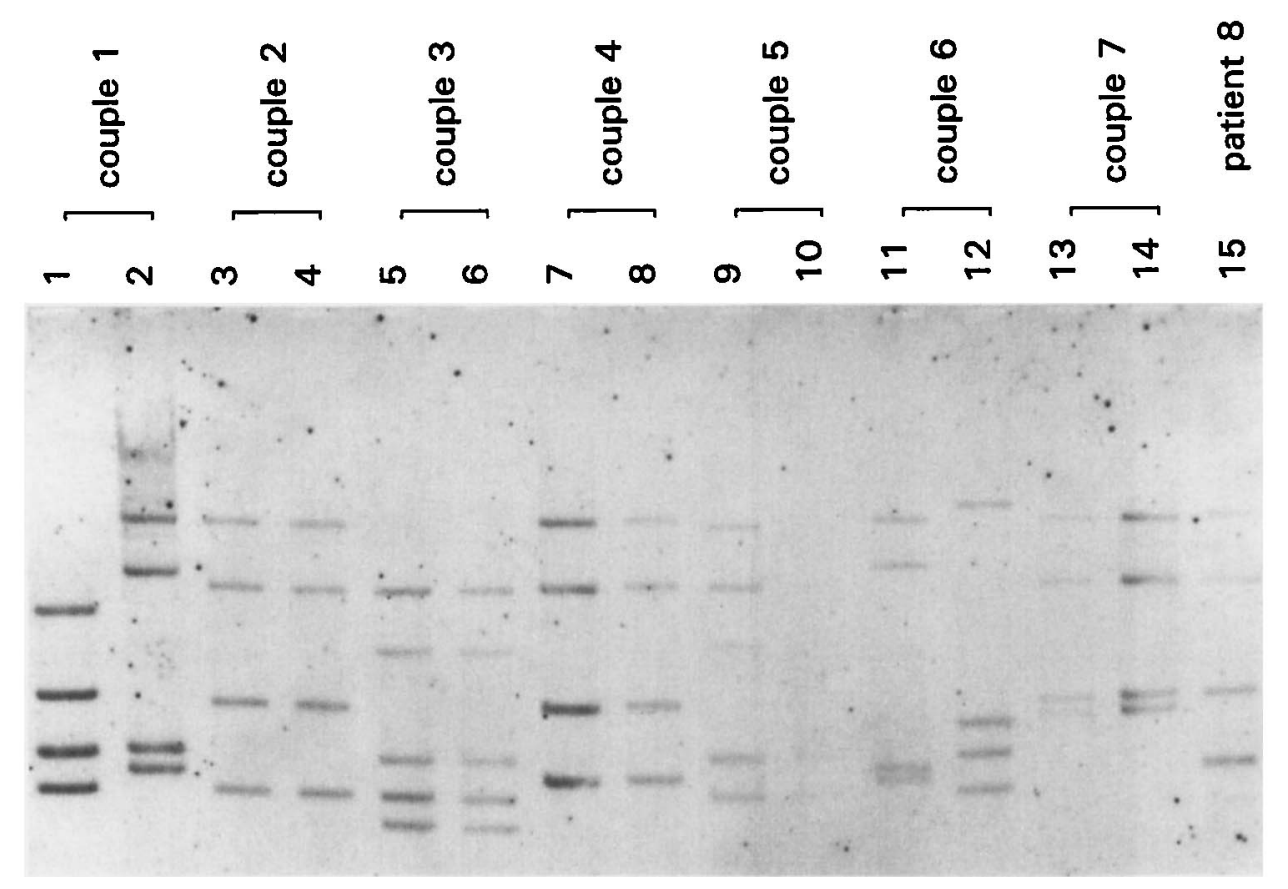

Fig. 3. Ribotypes visualised after BamHI digestion of DNA from 15 P. gingivalis isolates from eight married couples.

previously in these individuals. ${ }^{5}$ REA typing showed a total of 10 distinct types. Examples of the different REA types are shown in fig. 1 for six isolates from three married couples (BamHI digest); lanes 2 and 3 (couple 2) were indistinguishable, as were lanes 4 and 5 (couple 4); the two isolates from couple 6 were distinct (lanes 6 and 7). The clearest differences in the banding patterns were found in the molecular size region above $5 \mathrm{~kb}$. In two couples ( 3 and 8 ), minor differences indicative of subtypes were found between different isolates (results not shown). Isolates from unrelated individuals all had distinct DNA patterns, whereas the partners in six couples shared indistinguishable REA types. Isolates from different sites of a single individual gave indistinguishable patterns (table; couples 5 and 7). The data obtained by REA typing were in agreement with earlier results. ${ }^{\mathbf{5}}$

Typing by AP-PCR was evaluated with the same set of isolates. Fig. 2 shows the AP-PCR patterns obtained with primers $970-11$ and $910-05$ for 16 isolates of $P$. gingivalis, one from each individual. The pattern obtained with primer 970-11 for ATCC strain 33277 was similar to that reported in an earlier study, ${ }^{12}$ indicating good inter-assay reproducibility. Isolates from unrelated individuals gave distinct patterns, whereas those from six of the eight couples yielded indistinguishable DNA patterns, irrespective of the primer used. The banding patterns obtained with primer 910-05 were usually more complex than with primer 970-11, but allowed the same discrimination. The size of the PCR amplicons with both primers ranged from $0 \cdot 3$ to $2 \mathrm{~kb}$. The AP-PCR and REA typing data were in agreement with respect to the main types found (table). The subtypes differentiated by REA (on the basis of minor differences in the high molecular size region of the DNA fingerprint) could not be distinguished by AP-PCR.

Ribotyping was performed after digestion of chromosomal DNA with restriction endonucleases $B g l \mathrm{I}$ or $B a m \mathrm{HI}$, followed by hybridisation of the digests with ribosomal DNA from $E$. coli. Fig. 3 shows examples of the ribotypes obtained for 15 isolates after digestion with BamHI. The patterns obtained after digestion with $B a m H I$ were more distinct than those obtained after digestion with $B g l$ (not shown). The size of the DNA fragments identified by ribotyping ranged from 5 to $20 \mathrm{~kb}$. Ribotyping with both enzymes identified 11 distinct patterns. The ribotyping results were largely in agreement with the results obtained by REA typing and AP-PCR. As with AP-PCR, ribotyping was not able to discriminate between the strains with minor differences observed by REA typing in couples 3 and 8 (table). These differences in the REA pattern were observed after digestion with BamHI, electrophoresis and staining with ethidium bromide. After blotting of the same gel, no differences were found after hybridisation of the fragments with rDNA.

All three methods were reproducible. This was illustrated by the finding that all seven isolates from different sites of the patient in couple 5 were indistinguishable by all three methods. With typing by REA and AP-PCR, all isolates tested from couple 7 gave indistinguishable patterns; in contrast, ribotyping of these isolates showed heterogeneity: isolate HG 1352 was distinct from the other seven isolates after digestion with $B g I$, and isolate $H G 1354$ was distinct after digestion with BamHI (table). Fig. 4a shows that the REA patterns from isolates HG 1354 and HG 1359 were indistinguishable, whereas hybridisation with rDNA of the fragments from the same 


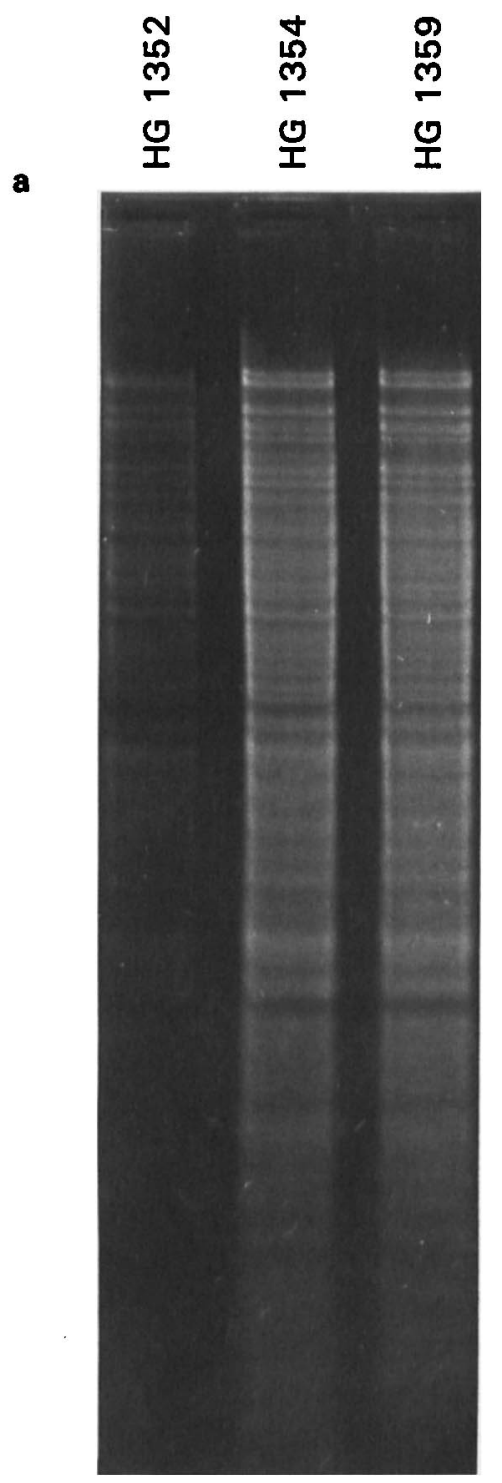

gel showed a clearly distinct pattern for HG 1354 (fig. $4 b)$. These results were found to be reproducible in three separate experiments.

\section{Discussion}

Several conventional typing methods, including biotyping, serotyping and antibiogram typing, have been described for $P$. gingivalis, ${ }^{14,15}$ but these methods recognise insufficient heterogeneity between strains to study transmission routes of this suspected periodontal pathogen. New molecular typing methods for bacteria have recently been applied to $P$. gingivalis. REA typing of $P$. gingivalis ${ }^{7^{-9}}$ is highly sensitive and reveals considerable heterogeneity between strains, but produces large numbers of DNA fragments, resulting in patterns which can be difficult to interpret when large numbers of isolates have to be evaluated. In contrast, ribotyping ${ }^{16}$ results usually in only four to eight bands. This method has been applied to $P$. gingivalis isolates from six ${ }^{11}$ and nine ${ }^{10}$ unrelated subjects, all of which

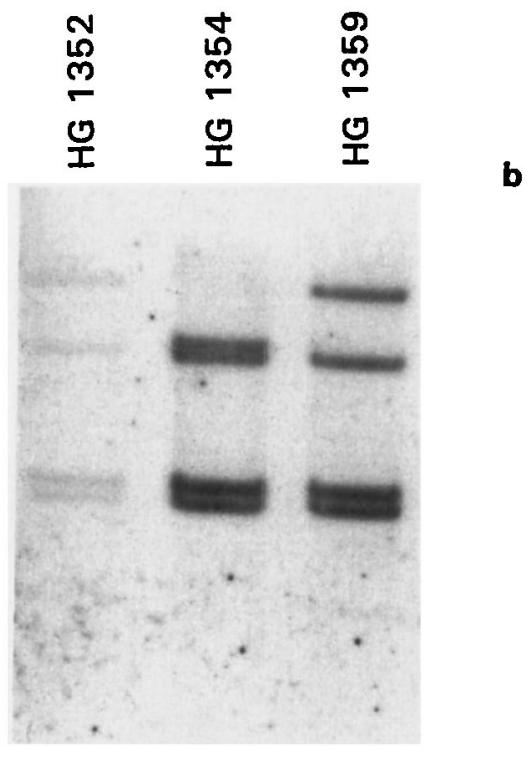

Fig. 4. a, Restriction endonuclease pattern after BamHI digestion; $b$, ribotype pattern from the same gel of three isolates from couple 7 .

were found to be distinct. Random amplification of DNA by PCR with an arbitrary primer (AP-PCR) can also be used for typing bacteria. ${ }^{17,18}$ Random amplification of genomic DNA, directed by a single oligonucleotide of arbitrary sequence, produces a set of varying short DNA products. Menard et al. ${ }^{12}$ applied this method to $P$. gingivalis and found that nine strains yielded nine distinct banding patterns. The present study compared these three typing methods with a set of $32 P$. gingivalis isolates from eight periodontitis patients and their spouses.

All three methods demonstrated considerable heterogeneity in $P$. gingivalis, observed as DNA fragment length polymorphisms, with 10 main types and three additional subtypes by REA, 10 types by AP-PCR, and 11 distinct types by ribotyping. The epidemiological conclusions arrived at by the three methods largely agreed: in six couples, the $P$. gingivalis types found in husband and wife were indistinguishable; in two couples ( 1 and 6 ), isolates from husband and wife were clearly distinct; and isolates from unrelated individuals, i.e., from different couples, were always 
distinct. Indistinguishable patterns obtained with isolates from different sites in the same individual were indicative of monoclonal colonisation. These data strengthen the conclusion, based on previous experiments with the same set of strains, that $P$. gingivalis can probably be transmitted between spouses. ${ }^{5}$

Relatively few differences were observed between the results obtained with the three methods. The minor differences between some strains found by REA (type $\mathrm{D}^{\prime}, \mathrm{L}^{\prime}$ and $\mathrm{L}^{\prime \prime}$ ), observed as a variable banding pattern in the high molecular size region, were not reflected in differences by AP-PCR or ribotype. This suggests that such minor polymorphisms reflect mutations restricted to a small part of the genome of these isolates. Such mutations outside the primer-target pairing site are overlooked by AP-PCR. A further discrepancy between the three methods was found with some isolates from couple 7 . One of eight isolates tested was distinct from the other seven isolates after ribotyping with $B g I I$, and a second isolate was distinct after ribotyping with $B a m \mathrm{HI}$. As this discrimination was not obtained by REA or AP-PCR, it can be speculated that these differences in ribotype represent relatively small polymorphisms in the genome of these isolates. This would imply that clear differences in ribotype among strains do not necessarily mean that the isolates are unrelated,

\section{References}

1. Mayrand D, Holt SC. Biology of asaccharolytic blackpigmented Bacteroides species. Microbiol Rev 1988; 52: $134-152$.

2. Slots J, Listgarten M. Bacteroides gingivalis, Bacteroides intermedius and Actinobacillus actinomycetemcomitans in human periodontal diseases. J Clin Periodontol 1988; 15: 85-93.

3. van Steenbergen TJM, van Winkelhoff AJ, de Graaff J. Blackpigmented oral anaerobic rods: classification and role in periodontal disease. In: Hamada S, Holt HC, McGhee JR (eds) Periodontal disease: pathogens and host immune responses. Tokyo, Quintessence Publishing Co. 1991: 41-52.

4. van Winkelhoff AJ, van Steenbergen TJM, de Graaff J. The role of black-pigmented Bacteroides in human oral infections. $J$ Clin Periodontol 1988; 15: 145-155.

5. van Steenbergen TJM, Petit MDA, Scholte LHM, van der Velden U, de Graaff J. Transmission of Porphyromonas gingivalis between spouses. J Clin Periodontol 1993; 20: 304-345.

6. Petit MDA, van Steenbergen TJM, Scholte LHM, van der Velden U, de Graaff J. Epidemiology and transmission of Porphyromonas gingivalis and Actinobacillus actinomycetemcomitans among children and their family members. A report of 4 surveys. J Clin Periodontol 1993 (in press).

7. Loos BG, Mayrand D, Genco RJ, Dickenson DP. Genetic heterogeneity of Porphyromonas (Bacteroides) gingivalis by genomic DNA fingerprinting. $J$ Dent Res 1990; 69: 1488-1493.

8. van Steenbergen TJM, van Winkelhoff AJ, van der Velden U, de Graaff J. Taxonomy, virulence and epidemiology of blackpigmented Bacteroides species in relation to oral infections. Infection 1989; 17: 194-196. and that minute differences can be detected even if their genomes have a high degree of similarity when measured by other techniques.

Since all three methods demonstrated considerable heterogeneity among independently obtained strains of $P$. gingivalis, it is questionable whether any of these methods is useful to study genetic relationships and to make correlations with virulence and pathogenicity. However, all three methods are suitable for studies of the routes of transmission, although AP-PCR may offer some advantages over the other two methods. REA typing provides a complex pattern with a large number of visible bands (usually $>30$ ), which makes comparison of lanes difficult, whereas ribotyping and AP-PCR both result in a limited number of bands, rendering comparison easier. A disadvantage of ribotyping is that it is rather time-consuming, in contrast to AP-PCR which is relatively easy to perform, gives reproducible data in less than $24 \mathrm{~h}$, and can be applied to a single colony. We conclude that AP-PCR may be of great value for epidemiological studies of $P$. gingivalis, and may also be useful for studies of other bacteria.

This work was supported in part by a grant from the Netherlands Organization for Scientific Research (NWO).

9. van Steenbergen TJM, van der Velden U, Abbas F, de Graaff J Microflora and bacterial DNA restriction enzyme analysis in young adults with periodontitis. J Periodontology 1991 ; 62: $235-241$

10. Saarela M, Stucki AM, von Troil-Linden B, Alalusua S, Jousimies-Somer $\mathrm{H}$, Asikainen $\mathrm{S}$. Intra- and interindividual comparison of Porphyromonas gingivalis genotypes. FEMS Immunol Microbiol 1993; 6: 99-102.

11. Socransky SS, Martin L. Ribotyping of $P$. gingivalis and $C$. rectus. J Dent Res 1992; $71: 246$ (abstract).

12. Ménard C, Brousseau R, Mouton C. Application of polymerase chain reaction with arbitrary primer (AP-PCR) to strain identification of Porphyromonas (Bacteroides) gingivalis. FEMS Microbiol Lett 1992; 95 : 163-168.

13. Brosius J, Ullrich A, Raker MA et al. Construction and fine mapping of recombinant plasmids containing the $\mathrm{rrnB}$ ribosomal RNA operon of E. coli. Plasmid 1981; 6: 112-118.

14. Loos BG, Dyer DW, Genco RJ, Selander RK, Dickinson AP. Natural history and epidemiology of Porphyromonas gingivalis. In: Shah HN, Mayrand D, Genco RJ (eds) Porphyromonas gingivalis. Boca Raton, FL, CRC Press Inc. 1993 (in press).

15. van Steenbergen TJM, van Winkelhoff AJ, de Graaff J. Classification and typing methods of black-pigmented Gram-negative anaerobes. FEMS Immunol Microbiol $1993 ; 6: 83-88$.

16. Grimont F, Grimont PAD. Ribosomal ribonucleic acid gene restriction patterns as potential taxonomic tools. Ann Inst Pasteur/Microbiol 1986; 137B: 165-175.

17. Welsh J, McClelland M. Fingerprinting genomes using PCR with arbitrary primers. Nucleic Acids Res 1990; 18: 7213-7218.

18. Williams GK, Kubelik AR, Livak KJ, Rafalski JA, Tingey SV. DNA polymorphisms amplified by arbitrary primers are useful as genetic markers. Nucleic Acids Res 1990; 18: 6531-6535. 